\title{
SIMULATIONS OF SOLENOID AND ELECTROSTATIC QUADRUPOLE FOCUSING OF HIGH INTENSITY BEAMS FROM ECR ION SOURCE*
}

\author{
Q. Zhao", A. Balabin, M. Doleans, F. Marti, J. W. Stetson, X. Wu, MSU, East Lansing, MI 48842, \\ U.S.A.
}

\section{Abstract}

Because of its axisymmetric property and reasonable effectiveness, solenoids have been widely used to focus beams at various injectors. For a multi-component beam with solenoidal focusing, beam quality can be significantly deteriorated due to the magnetic focusing strength dependence on the beam charge-to-mass ratio. Electrostatic quadrupole focusing has been explored as an alternative option at the National Superconducting Cyclotron Laboratory (NSCL) for the injection line of the superconducting cyclotrons. We present the results of simulations for both systems.

\section{INTRODUCTION}

Electron Cyclotron Resonance (ECR) ion sources provide high current ion beams for injection into the two coupled superconducting cyclotrons at the NSCL. Since the extracted beam from the ECR usually consists of ions with a broad range of charge states, the total beam intensity is much higher than the single charge state beam of interest. Proper initial focusing outside the ECR must be provided to control beam envelope and emittance growth due to space charge effects and to match the beam into the analysis magnet for charge state selection. A solenoid has originally been used at the NSCL with reasonable success as the initial focusing element for the multi-component beam extracted from an ECR. However, the beam was found to have a significantly hollow transverse current profile and injection efficiency into the coupled cyclotrons was relatively low, although the beam transport efficiency in the injection line was high [1]. Primarily to avoid the focal length charge-to-mass dependence of the solenoids, electrostatic quadrupoles were explored as an alternative option. The layout of one injection beam line is shown in Fig. 1.

Six-dimensional particle tracking of multiple charge state beams including the effects of space-charge from the ECR plasma chamber through aperture 2 of Fig. 1 was done. KOBRA [2] and PARMELA [3] both of which include full space charge effects and use threedimensional electromagnetic field models for the focusing elements were the main simulation codes employed.

\section{BEAM FORMATION}

KOBRA was used to simulate beams from the ECR plasma chamber through the extraction system to a point where the ECR solenoid magnetic field is negligible ( $\sim 30 \mathrm{~cm}$ away from ECR extraction aperture). An argon beam was simulated with an ECR extraction voltage of

\footnotetext{
*Supported by National Science Foundation under grant PHY-0110253. \# zhao@nscl.msu.edu
}

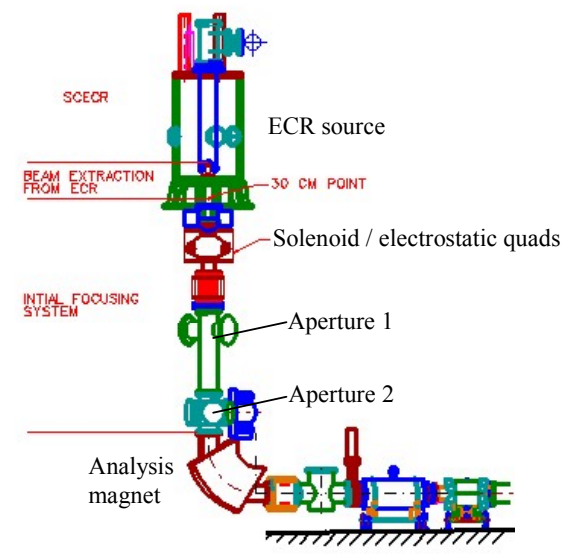

Figure 1: Layout of injection beam line

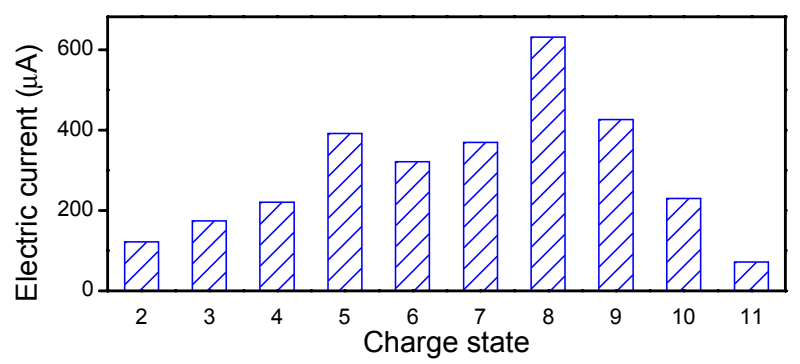

Figure 2: Charge state distribution spectrum of argon beam used in simulations.
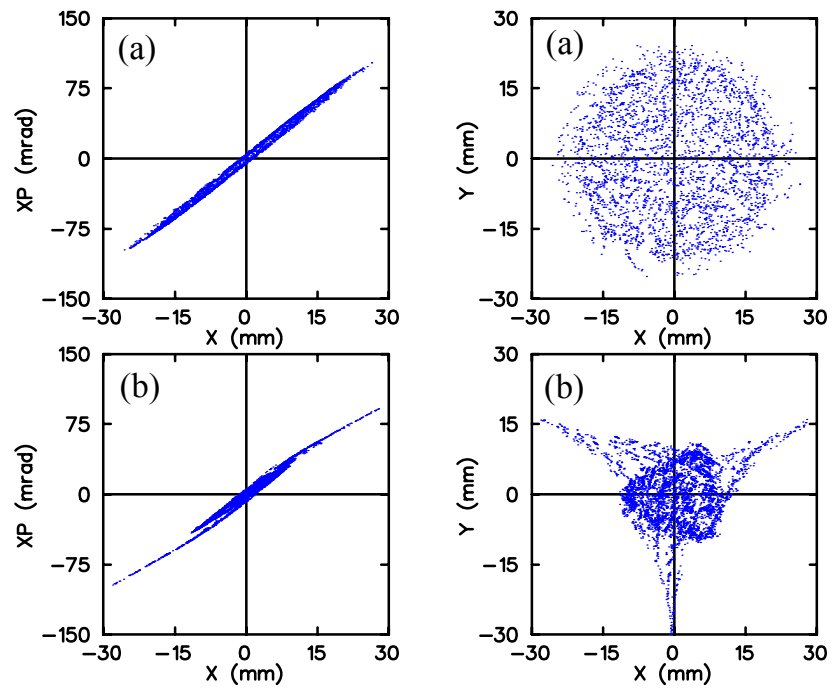

Figure 3: Transverse phase space of ${ }^{40} \mathrm{Ar}^{7+}$ beam at $30 \mathrm{~cm}$ from ECR extraction with two different initial particle distributions in the plasma chamber. The (a) and (b) panels are the first and second cases respectively described in the text. (Other charge-state beams are not shown here but were included in the simulations). 
$24.5 \mathrm{kV}$ and a total beam current including all charge states of about $3 \mathrm{~mA}$. Ten charge states of argon with a distribution based a on measured spectrum were included in the simulations as shown in Fig. 2. Two different initial particle distributions were used. The first one shown in panel (a) of Fig. 3 assumed ions of different charge states were uniformly populated near the ECR extraction aperture and did not include the ECR sextupole fields. The second one shown in panel (b) of Fig. 3 assumed the ions were randomly distributed in a volume inside the ECR plasma chamber whose surface was determined by the electron resonance condition with the radial distributions adjusted to obtain similar output normalized emittances for the different charge states. The full beam emittance for both cases was $\sim 200 \pi \mathrm{mm}$-mrad. The second model including the ECR sextupole field produces the observed triangular beam pattern and will be used in future evaluations, but only the results of the downstream tracking using the first case have been completed at this juncture and are discussed here.

\section{INITIAL FOCUSING SYSTEM}

\section{Solenoid Focusing}

Originally used in all ECR initial focusing systems at the NSCL, a solenoid focusing system is simple and provides axially symmetric focusing for both transverse planes. A two-dimensional cylindrical field of a solenoid with a 540 turn coil, an iron yoke, and a bore radius of 5 $\mathrm{cm}$ was obtained using POISSON and used for particle tracking to evaluate aberration effects.
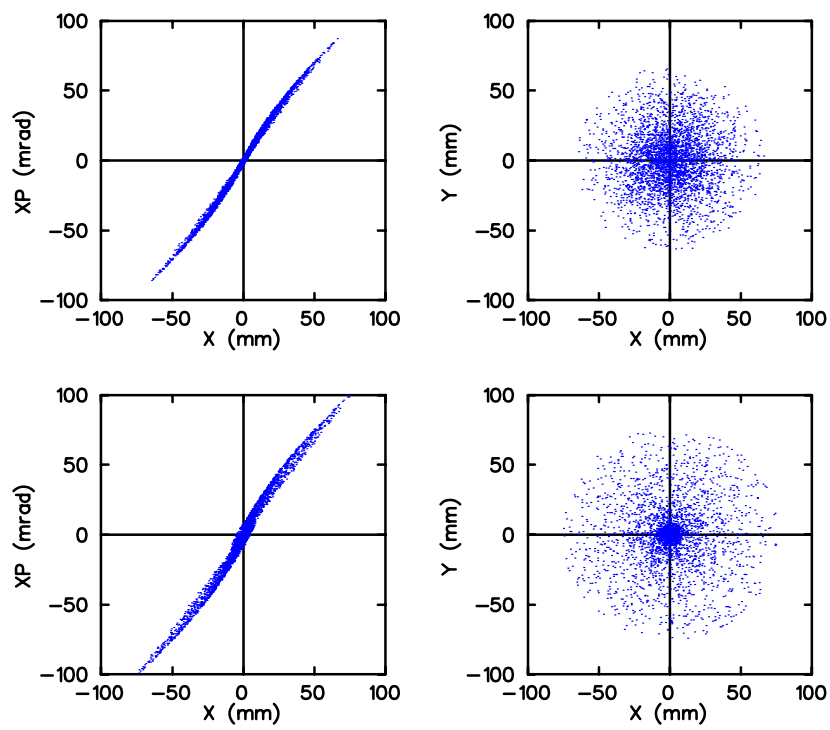

Figure $4:{ }^{40} \mathrm{Ar}^{7+}$ beam distributions after solenoid focusing for solenoid aberration only (top) and solenoid aberration with space charge effects (bottom).

Fig. 4 shows the particle tracking results of an ${ }^{40} \mathrm{Ar}^{7+}$ beam through a solenoid focusing system with and without space charge effects. Solenoid fields were adjusted to achieve similar beam Twiss parameters for the two cases. Without space charge, the phase space is slightly distorted due to aberrations of the solenoid field. When space charge effects are included, the phase space has significantly larger distortion and halo formation. The effect was quantified by calculating the percentage of the beam included in a given beam emittance as shown in Fig. 5. The beam brightness is reduced due to the aberrations in the solenoid field (red dashed). The largest reduction in brightness is obtained with the inclusion of space charge (blue dot). Given an estimated cyclotron beam acceptance, about 70,55 , and $30 \%$ of the beam would be injected for the case of no solenoid aberration, solenoid aberration, and aberrations with space charge respectively.

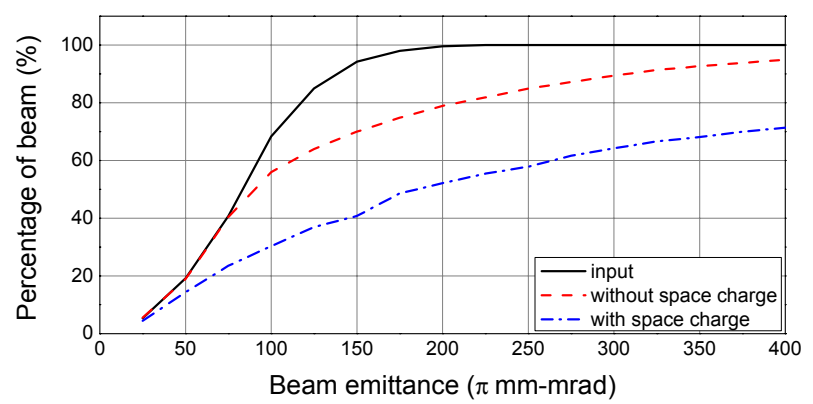

Figure 5: Percentage of included ${ }^{40} \mathrm{Ar}^{7+}$ beam vs. emittance in solenoid focusing system for no solenoid aberration (black solid), solenoid aberration only (red dash), and aberrations with space-charge effects (blue dot).

\section{Electrostatic Quadrupole Focusing}

To circumvent charge state focusing dependence of solenoids, a system using electrostatic quadrupoles was explored. Electrostatic quadrupoles provide stronger focusing than Einzel lens and unlike solenoid focusing, are independent of beam charge-to-mass ratio. However, several quadrupoles are necessary to provide axially symmetric focusing and the quadrupoles can have large aberrations causing an emittance growth. High-order correction may be necessary, especially for a large size beam [4].

\section{Electrostatic Quadrupole Triplet Focusing}

An electrostatic quadrupole triplet was purchased from National Electrostatics Corp. The lengths of the triplet electrodes were 5,10 , and $5 \mathrm{~cm}$ with a bore diameter of $76 \mathrm{~mm}$. A collimator with an aperture of $50 \mathrm{~mm}$ was installed in front of the triplet to protect the electrodes from beam. Three-dimensional fields of the triplet were developed for particle tracking. The voltages on the electrodes were adjusted so that the beam Twiss parameters were similar to those of the solenoid focusing system. Fig. 6 shows the distributions of the gradient of the transverse electric fields and the longitudinal electric fields at four different radial positions of the triplet $(0.25 \mathrm{R}, 0.5 \mathrm{R}, 0.75 \mathrm{R}$, and $0.95 \mathrm{R}$ for a bore radius of $\mathrm{R}=3.68 \mathrm{~cm}$ ). Ideally, the gradient of transverse electric fields should be constant, independent of radius. However, due to the low ratio of length-to-aperture, no flatness of the fields is achieved for the two outside quads. 
In addition, there are strongly radial dependent longitudinal fields in the fringe regions that would accelerate or decelerate off-axis particles leading to additional aberrations.

Particle tracking results of an ${ }^{40} \mathrm{Ar}^{7+}$ beam through an electrostatic triplet focusing system with and without space charge effects are shown in Fig. 7. The "s-shaped" phase space is due primarily to the triplet aberrations. Again the percentage of the beam included in a given beam emittance was calculated as shown in Fig. 8. For this case the dominant effect was due to field aberrations of triplet exacerbated since the incident beam filled the whole aperture.
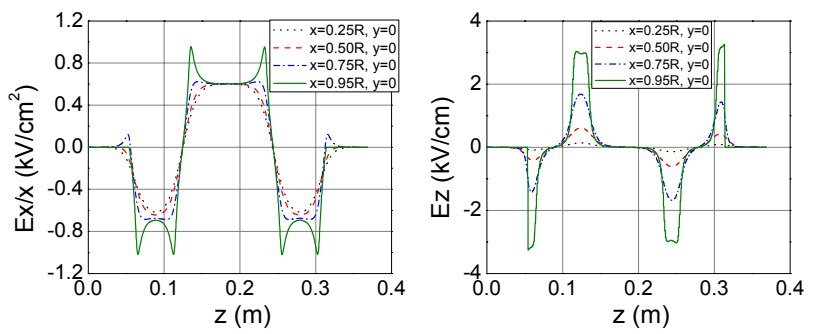

Figure 6: Distributions of the gradient of the transverse electric fields (left) and the longitudinal electric fields (right) at four different radial positions of the triplet.
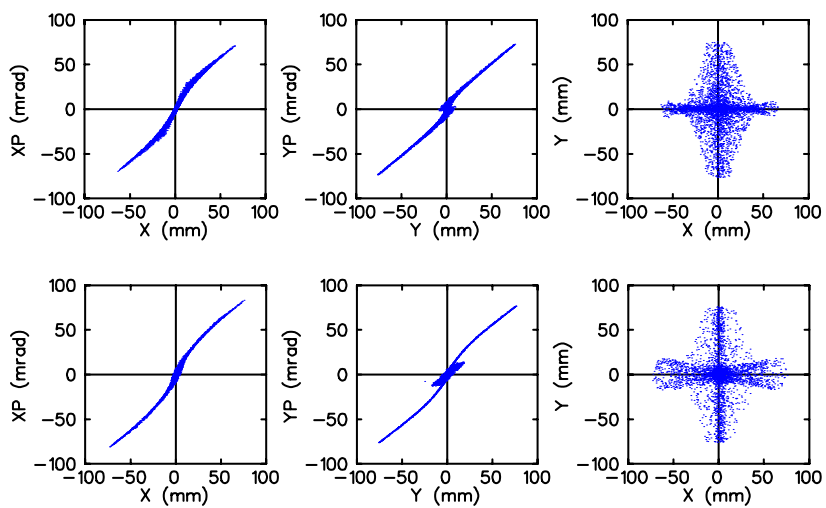

Figure 7: ${ }^{40} \mathrm{Ar}^{7+}$ beam distributions after electrostatic triplet focusing for field aberrations only (tops) and field aberration with space charge effects (bottoms)

Simulation results indicate that a solenoid provides better performance than an electrostatic quadrupole triplet (compare Fig. 5 and Fig. 8). Given an estimated cyclotron beam acceptance, about $30 \%$ vs. $20 \%$ of the beam would be injected with solenoid focusing and electrostatic quadrupole triplet focusing, respectively. However, this is inconsistent with experimental observations that show an efficiency gain with an electrostatic quadrupole triplet focusing system over that of a solenoid. The discrepancy between simulation and experiment may be caused by the initial beam conditions used in the simulations. For example, the dominant effect of field aberration for the case of the electrostatic quadrupole triplet is caused by the large beam size at that point resulting from the assumed initial beam conditions. Both simulation and experiment show no hollow beam formation with electrostatic quadrupole triplet focusing. Experimental emittance measurement studies using a newly constructed emittance scanner downstream of the analysis magnet are underway to obtain a better understanding of the different focusing systems.

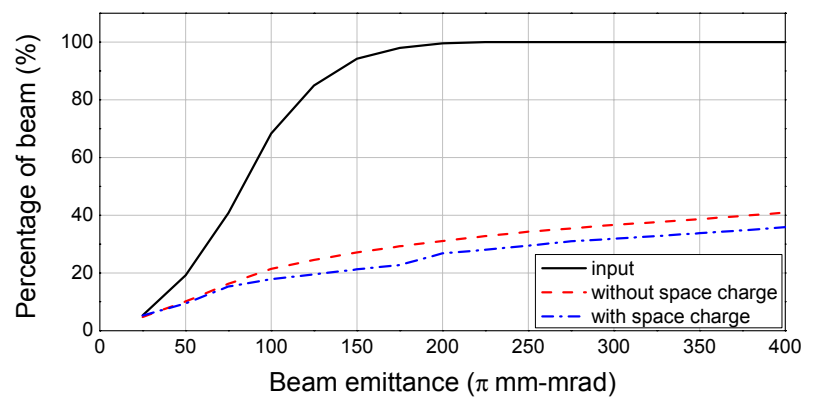

Figure 8: Percentage of included ${ }^{40} \mathrm{Ar}^{7+}$ beam vs. emittance in electrostatic triplet focusing system for input beam (black solid), triplet aberrations only (red dash), and triplet aberrations with space-charge effects (blue dot).

\section{Electrostatic Quadrupole Doublet Focusing}

An electrostatic quadrupole doublet focusing system was proposed to upgrade the existing triplet for further performance improvement. The system has larger bore radius to increase beam transmission, optimized system geometric layout, and possible high order corrections to minimize the aberration effects [4]. Furthermore, it can have a transverse phase advance of $\pi$, that may compensate the beam distortions due to the sextupole component originated from ECR sextupole fields with an external sextupole magnet.

\section{SUMMARY}

Particle tracking studies were performed including three-dimensional electromagnetic fields and space charge effects for high current, multi-charge state ion beams extracted from an ECR ion sources at the NSCL. The results show space charge effects have significant impact on beam emittance in solenoid focusing, while the impact is dominated by aberrations in electrostatic quadrupole triplet system. Further detailed comparisons between simulation and measurement are planed. A focusing system of four electrostatic quadrupoles has been proposed to further improve performance.

\section{REFERENCES}

[1] J. W. Stetson, G. Machicoane, F. Marti, P. Miller, M. Steiner, P. Zavodzsky and N.Y. Kazarinov, "A Comparison of Electrostatic and Magnetic focusing of Mixed Species Heavy Ion Beams at NSCL/MSU", these proceedings.

[2] P. Spaedtke, "KOBRA3-INP", 2005.

[3] L. M. Young, "PARMELA", Los Alamos National Laboratory report LA-UR-96-1835 (Revised April 26, 2005).

[4] R. Baartman, "Aberrations in Electrostatic Quadrupoles", TRI-DN-95-21, TRIUMF, June, 1995. 